\title{
Investigation and identification of functional post-translational modification sites associated with drug binding and protein-protein interactions
}

\author{
Min-Gang Su ${ }^{1 \dagger}$, Julia Tzu-Ya Weng ${ }^{1 \dagger}$, Justin Bo-Kai Hsu ${ }^{2+}$, Kai-Yao Huang ${ }^{1,3}$, Yu-Hsiang Chi ${ }^{1}$ and Tzong-Yi Lee ${ }^{1,4^{*}}$
}

From 16th International Conference on Bioinformatics (InCoB 2017)

Shenzhen, China. 20-22 September 2017

\begin{abstract}
Background: Protein post-translational modification (PTM) plays an essential role in various cellular processes that modulates the physical and chemical properties, folding, conformation, stability and activity of proteins, thereby modifying the functions of proteins. The improved throughput of mass spectrometry (MS) or MS/MS technology has not only brought about a surge in proteome-scale studies, but also contributed to a fruitful list of identified PTMs. However, with the increase in the number of identified PTMs, perhaps the more crucial question is what kind of biological mechanisms these PTMs are involved in. This is particularly important in light of the fact that most protein-based pharmaceuticals deliver their therapeutic effects through some form of PTM. Yet, our understanding is still limited with respect to the local effects and frequency of PTM sites near pharmaceutical binding sites and the interfaces of protein-protein interaction (PPI). Understanding PTM's function is critical to our ability to manipulate the biological mechanisms of protein.
\end{abstract}

Results: In this study, to understand the regulation of protein functions by PTMs, we mapped 25,835 PTM sites to proteins with available three-dimensional (3D) structural information in the Protein Data Bank (PDB), including 1785 modified PTM sites on the 3D structure. Based on the acquired structural PTM sites, we proposed to use five properties for the structural characterization of PTM substrate sites: the spatial composition of amino acids, residues and side-chain orientations surrounding the PTM substrate sites, as well as the secondary structure, division of acidity and alkaline residues, and solvent-accessible surface area. We further mapped the structural PTM sites to the structures of drug binding and PPI sites, identifying a total of 1917 PTM sites that may affect PPI and 3951 PTM sites associated with drug-target binding. An integrated analytical platform (CruxPTM), with a variety of methods and online molecular docking tools for exploring the structural characteristics of PTMs, is presented. In addition, all tertiary structures of PTM sites on proteins can be visualized using the JSmol program.

Conclusion: Resolving the function of PTM sites is important for understanding the role that proteins play in biological mechanisms. Our work attempted to delineate the structural correlation between PTM sites and PPI or drug-target binding. CurxPTM could help scientists narrow the scope of their PTM research and enhance the efficiency of PTM identification in the face of big proteome data. CruxPTM is now available at http://csb.cse.yzu.edu.tw/CruxPTM/.

\footnotetext{
* Correspondence: francis@saturn.yzu.edu.tw

${ }^{\dagger}$ Equal contributors

'Department of Computer Science and Engineering, Yuan Ze University,

Taoyuan 320, Taiwan

${ }^{4}$ Innovation Center for Big Data and Digital Convergence, Yuan Ze University,

Taoyuan 320, Taiwan

Full list of author information is available at the end of the article
} 


\section{Background}

Proteins are the major functional molecules in living cells, playing essential roles in various cellular processes such as catalysis, transport, and structural integrity. Although the human genome is estimated to harbor approximately 25,000 genes [1], alternative splicing of transcripts and post-translational modifications (PTMs) of proteins result in millions of proteins with diverse functions [2, 3]. PTMs regulate a protein's function, level, and activity through the covalent attachment of small chemical molecules to certain amino acid residues, allowing proteins to respond to developmental signals or environmental stimuli [4,5]. A protein's structure can also be altered by these site-specific chemical modifications, leading to changes in stability, localization, and associations with other interacting molecules [6].

Recent advancement in high-throughput mass spectrometry (MS)-based proteomics technology has facilitated the identification of more than 200 different PTMs [7]. Many databases [6, 8-14] and tools [15-31] have been proposed for characterizing and identifying the substrate sites of a specific PTM type. Regarding the structural investigation of PTM sites, Zanzoni et al. have built a database of three-dimensional structures of protein phosphorylation sites (Phospho3D) in 2007 [15]. As an update to Phospho3D [16] published in 2011, Su et al. made a successful attempt at uncovering kinaseassociated phosphorylation sites on the three-dimensional structure of proteins, by incorporating information such as spatial amino acid composition and substrate sequence motifs [17]. In 2014, Su et al. developed a new resource (topPTM) that considers transmembrane topology on 3D structures for the identification of functional PTM sites on membrane proteins [18]. Additionally, Craveur et al. designed a database (PTM-SD) for accumulating structurally resolved PTMs in proteins [19]. Although several databases were dedicated to characterizing the structures of PTM sites on protein tertiary structures, there exists no resource currently for providing an integrative platform to explore the PTM sites associated with drug binding and protein-protein interaction. According to the PTM data presented in UnitProtKB [20] and dbPTM [6], Table 1 shows the data statistics of PTM sites involved in protein-

Table 1 Number of PTM proteins associated with PPI and drug binding

\begin{tabular}{lll}
\hline & All proteins & Human proteins \\
\hline Total proteins & 550,299 & 20,186 \\
PTM proteins & 112,985 & 13,080 \\
PPI annotation (string) & 326,227 & 17,833 \\
PTM \& PPI proteins & 65,907 & 12,541 \\
Drug-binding proteins(DrugBank) & - & 1980 \\
PTM \& drug-binding proteins & - & 1404 \\
\hline
\end{tabular}

protein interaction (PPI), including drug binding. It appears that at least $65 \%(13,080 / 20,186)$ of known human proteins are regulated by PTM. More than $70 \%$ of human proteins can undergo PTM and may interact with other proteins. At the same time, over $70 \%$ of human proteins represent drug targets and can be altered by PTM. Therefore, PTM may be highly correlated with drug binding and PPI.

Indeed, an increasing number of studies are uncovering evidence of PTMs regulating drug-target interactions. For example, the epigenetic regulation of the chaperone cycle in different cell types or environmental conditions is found to involve changes in Hsp90 (heat shock protein 90) function through PTM [21]. Moreover, it has been shown that the effect of Hsp90 inhibitors could be enhanced when enzymes that facilitate the PTM of Hsp90 were suppressed [22], lending support to PTM being a potential therapeutic strategy for modulating the activity of Hsp90 in cancer cells. Phosphorylation, a common PTM of proteins, has also been utilized in drug-target design, whereby the interaction between the drug and the target is controlled by the state of phosphorylation [23, 24]. For instance, it is suggested that various upstream activators and different phosphorylation states can have a spectrum of effects on MEK inhibition, and therefore, greatly influence drug-target interaction with respect to MEK kinase pathway [25]. Since a large proportion of proteins undergo PTMs, it is likely that changes in PTMs regulate a drug's efficacy and interaction with its target. Also, (Additional file 1: Table S1) shows that drugs can be categorized into two classes, small molecule drugs and biologics [26]. In general, most drugs are considered small organic compounds with a low molecular weight of less than $900 \mathrm{Da}$. Thus, for PTM studies in the context of drug-target binding, it would be reasonable to focus on the effects, frequency, and location of PTM near the site of binding by small molecule drugs.

The function of a protein can also be regulated by non-covalent PPIs [5, 27-31], a type of highly specific physical interactions between two or more protein molecules [32]. Many cellular processes are carried out through the complex interactions between various proteins, making up the interactome of a living cell or an organism. The binding affinities of these interacting proteins are also regulated by PTMs [5]. According to PTM data on the dbPTM database, more than $60 \%$ of PTM sites are found in the domains of proteins that actively participate in PPIs [18], providing support for a connection between PTM and PPI, and revealing the functions of the proteins involved in PPI. Thus, it is reasonable to assume that proteins capable of undergoing specific PTMs may exhibit certain properties related to PPI. 
The diverse effects of PTMs on proteins, as well as their regulatory functions in various cellular processes contributed to the focus of this study-the investigation of drug-target binding and PPIs associated with PTM sites. In particular, we integrated protein tertiary structure and PPI information with the associated PTM sites from the annotations of 3did (3D interacting domains) [33]. PTM peptides were manually curated, and based on their sequence identity with records in the Protein Data Bank (PDB) [34] and UnitProtKB ID, mapped to their associated proteins. To uncover the impact on binding attributable to residues structurally surrounding PTM substrate, we investigated the orientations of side chains encompassing these neighboring residues in relation to the location of the PTM substrate sites in a protein structure. Finally, we constructed a databaseassisted system, CruxPTM, to provide comprehensive information regarding PTM sites on protein tertiary structures, including the site-specific spatial composition of residues, surface area that is accessible to solvent, and residues that surround the PTM sites.

\section{Methods}

Figure 1 presents the workflow of this study. PTM sites that have been experimentally validated were acquired from dbPTM 3.0 [7], which is a useful database that comprehensively integrates all currently available PTM information. Since drug binding sites and proteinprotein interaction sites were extracted from protein structural information, we mapped all experimentally confirmed PTM sites to known 3D structures from the PDB for subsequent analyses. Next, the PTM sites were cross-matched with drug binding sites and PPI contacting sites for the identification of PTM sites associated

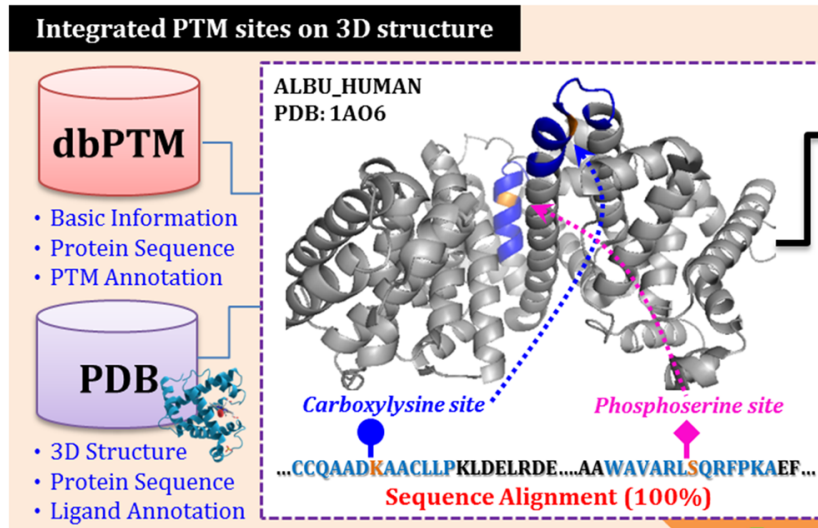

- Ligand Annotation Sequence Alignment (100\%)

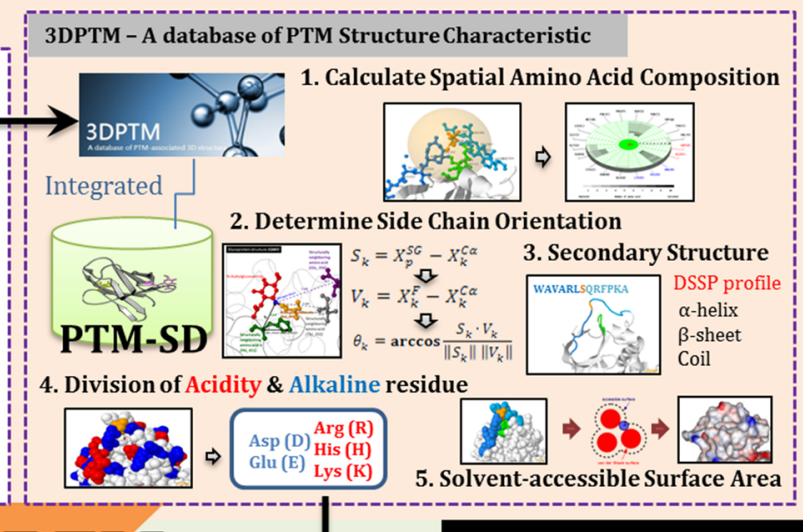

PTM associated with Drug Give PTM

PTM associated with PPI

Binding Energy Comparison between PTM \& Non-PTM..

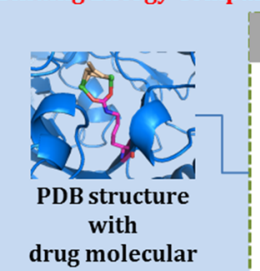

Experimental Drug Binding Sites
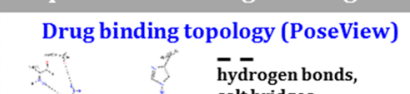

salt bridges,

metal interactions

hydrophobic interactions

$\pi-\pi$ and
$\pi-\operatorname{cation}$ inte

Cross-map to 3DPTM

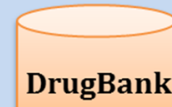

- Approved Drugs - Target Proteins

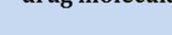

$\pi \cdot$ cation int

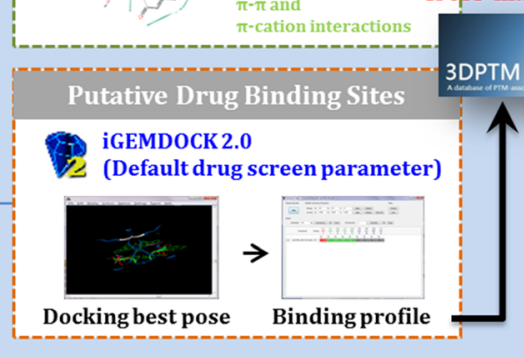

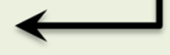
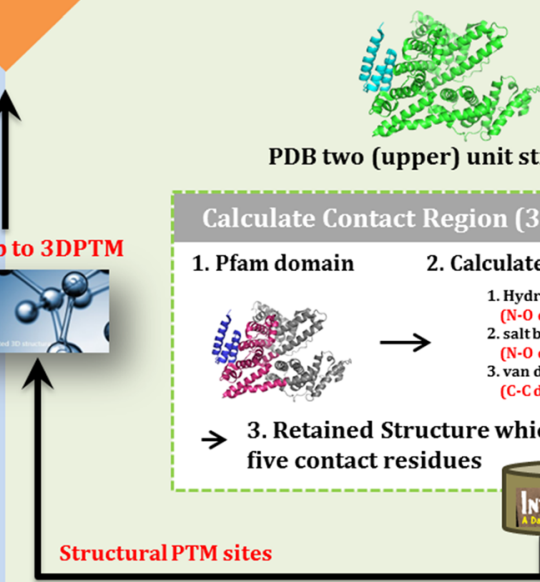

PDB two (upper) unit structure Calculate Contact Region (3DID method)

1. Pfam domain 2. Calculate contact residue

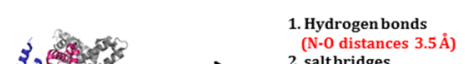
2. saltbridges $3.5 \AA$ ) (N-0 distances $5.5 \AA$ ) 3. van deWaals interactions
(C-C distances $5 \AA$ )

which more than

$\rightarrow$ 3. Retained Structure

Nteripry PPI contact residues

Fig. 1 Flowchart of the analyses performed in this study. The experimentally verified PTM sites were acquired from dbPTM. Since drug binding sites and protein-protein interaction sites were extracted from protein structural information, we mapped all experimentally confirmed PTM sites to known 3D structures in the PDB by using UniProtKB ID and sequence identity. Then, the PTM sites were cross-matched with drug binding sites and PPI contacting sites for the identification of PTM sites associated with drug binding and PPI. Finally, these data were integrated with a PTM structural analytical method and computing programs for building up a web-based system 
with drug binding and PPI. Finally, these data were combined with a PTM structural analytical method and computing programs for building up an online analysis platform. Detailed methods are as follows.

\section{Mapping of PTM sites to the tertiary structures of proteins}

To identify the spatial composition of PTM substrate sites within the tertiary structures of proteins, we obtained from the PDB protein structures that have been determined by NMR or X-ray crystallography with an experimental resolution less than $2.5 \AA$ [24]. According to the annotations in UniProtKB, 23,605 proteins in the PDB have 3D structure information. Also, chemical groups that can be covalently attached to the side chain of target residues were observed only in a few protein structures. Thus, to locate PTM substrate sites in 3D, mapping was performed between experimentally verified PTM peptides and the PDB protein records, and crossreferenced with the annotations of tertiary structures on UniProtKB with $100 \%$ similarity in sequence identity. Additionally, PTM sites possessing protein structures with modified residues were obtained from annotations on the PTM-SD database [19]. Most PTM sites that are mapped to structural sites are presented in the unmodified state, but PTM-SD provides complete information for modified PTM sites in 3D structures.

\section{Investigation of PTM sites associated with drugs binding}

While it is suggested that the binding affinity of a small molecule can be regulated by a phosphorylation site within $12 \AA$ of the site of binding [24], there is still a lack of information regarding the occurrences and influence of PTMs near drug-target binding. Therefore, we proposed a method in this study to identify PTM sites involved in drug binding. Figure 1 illustrates the workflow for extracting sites of drug-target binding in protein $3 \mathrm{D}$ structure. The entire process can be divided into two steps: 1) the processing of experimentally verified binding sites, and 2) molecular docking of drug binding. In step 1 , we collected the structural information of small molecules that have associated keywords such as "drug," "inhibitor," "agonist" or "antagonist" and have drug annotations in the DrugBank [35]. A total of 34,555 PDB structures and 4803 small drug molecules which have DrugBank annotations were obtained. Then, the PoseView [36] method was employed to check the binding sites of each drug in the target proteins. PoseView provides a twodimensional (2D) diagram showing how the drug ligand and the amino acid residues of the target protein may be arranged at the site of interaction. The nature of the interaction is presented in three ways. Black dashed lines indicate hydrogen bonds, salt bridges, and metal interactions. Green solid lines show hydrophobic interactions and green dashed lines represent $\pi-\pi$ and $\pi$-cation interactions.

In step 2, a docking program, iGEMDOCK 2.0 [37], was utilized for the computational extraction of drug binding sites. We followed the four sequential steps in iGEMDOCK to perform the drug-target interaction analysis: target and database preparations, molecular docking and post-docking analyses. First, coordinates of the target protein atoms acquired from PDB, the ligand binding area, the atom's formal charge and the atom types were specified. This procedure allowed iGEMDOCK to read the atom coordinates of a ligand from the prepared ligand database. After the ligand database and the target proteins were determined, docking was analyzed for each ligand using the flexible docking function provided by iGEMDOCK. The final step constituted the re-ranking and sorting of all docked ligand conformations based on an empirical scoring function and an evolutionary approach. The output of the program consisted of details regarding the docking result of each binding site, as well as the atomic characteristics of the target residues that interact with a specific drug ligand by hydrogen bonding $(\mathrm{H})$, electrostatic $(\mathrm{E})$ and van der Waal contact (V). A total of 1991 approved drugs from the DrugBank with 1632 target proteins were investigated by this proposed method. After mapping the experimentally verified PTM sites to the PDB structures, the PTM sites located in a drug binding site were determined to have strong associations with drug-binding, while those in the side chains that were within $12 \AA$ of a drug-binding site were considered to be have relatively weak association with drug binding.

\section{Identification of PTM sites related to protein-protein interactions}

In this work, the information of protein functional domains and PPIs were integrated for the identification of PTM-dependent protein interactions. To investigate the preferred functional domains of PTMs, we extracted the domain annotations from the Pfam database, which gives protein "signatures" based on protein families, domains and functional sites. In order to comprehensively study the structural properties of PTM sites associated with protein-interaction domains, the $3 \mathrm{D}$ structures of PPIs were acquired from the PDB. By adopting the 3D Interacting Domains (3DID) method proposed by Mosca et al. [33], the interaction interface of domain-domain interactions in the PDB 3D structures were determined as illustrated in Fig. 1. First we searched for protein structures with more than two subunits, and calculated the number of contact residues on the interface of the Pfam domain region containing the two subunits. Next, we applied a method based on previously published literature by Aloy and Russell [38], in which they derived 
the main-chain to side-chain and side-chain to sidechain potentials from the type of complexes described above. In particular, Aloy and Russell [38] defined interacting residues by using one or more of the following properties: hydrogen bonds (N-O distance of $3.5 \AA$ ), salt bridges (N-O distance of $5.5 \AA$ ), or van de Waals interactions (C-C distance of $5 \AA$ ). If there exists more than five pairs of residue contacts between two domains of a two-subunit region, these two subunits would be defined as an interaction structure. The contact residues were also extracted. A total of 30,455 PDB structures and 13,645 proteins were analyzed and 15,124 proteinprotein interaction pairs were defined. Mapping between the experimentally verified PTM sites and the PDB structure uncovered PTM sites located on the PPI interfaces. These sites were regarded as PTM-driven PPIs.

\section{Results and discussion}

\section{PTM substrate site characterization}

The availability of high-throughput proteomic technology has stimulated interests in understanding the structural environment of PTM substrate sites [17, 39]. To characterize PTM substrate sites, we considered a fivestep approach, focusing on protein properties such as spatial amino acid composition, structurally neighboring residues and side chain orientations surrounding the PTM substrate sites, as well as the secondary structure, division of acidity and alkaline residues, and solventaccessible surface area. In particular, we adopted the dictionary of protein secondary structure (DSSP) [40] for the calculation of solvent-accessible surface areas of proteins and for the standardization of PDB secondary structures with the corresponding PTM sites. To overcome difficulties that may arise during the derivation of substrate motifs from linear sequences [41], a radial cumulative propensity plot [42] was used to display the spatial composition and abundance of amino acids within and surrounding a particular PTM site (Fig. 2a). After a comprehensive and systematic analysis on the PDB structures, the number of PTM sites that can be mapped on protein structures is presented in (Additional file 2: Table S2). The spatial amino acid compositions were obtained by computing the relative frequencies of the 20 amino acids within 2 to $12 \AA$ radial distances of the modified residues. Next, using JSmol software [43], neighboring amino acids at the sequence level and in the spatial context were presented with different colors on the PDB 3D structures for the structural characterization of PTM substrate sites. Following the method of Ruzza et al., the functional roles and drug binding effects associated with a PTM substrate site's spatially neighboring residues were determined on the basis of these amino acids' side chain orientations. As shown in Fig. 2b, given an $\mathrm{N}$-linked glycosylation substrate site and its spatially neighboring amino acid, the vector from the residue to the nitrogen of $\mathrm{N}$-linked glycosylated asparagine $(p)$ is:

$$
S_{k}=X_{p}^{S G}-X_{k}^{C \alpha}
$$

where the crystallographic positions of the nitrogen in glycosylated asparagine $p$ and the $C_{\alpha}$ atom in residue $k$ are represented by $X_{p}^{S G} \mathrm{p}$ and $X_{k}^{C \alpha}$, respectively. The vector $V_{k}$ defines the direction of the side chain of a spatially neighboring amino acid $k$ from its $C_{\alpha}$ atom to the functional atom (58):

$$
V_{k}=X_{k}^{F}-X_{k}^{C \alpha}
$$

where $X_{k}^{F}$ is the crystallographic positions of the functional atom, while $X_{k}^{C \alpha}$ is the $C_{\alpha}$ atom in residue $k$. The effect of the side chain of a spatially neighboring amino acid, $k$, on the substrate asparagine residue is defined by the angle $\theta_{k}$ between vectors $S_{k}$ and $V_{k}$ :

$$
\theta_{k}=\arccos \frac{S_{k} \cdot V_{k}}{\left\|S_{k}\right\|\left\|V_{k}\right\|}
$$

The angle $\theta_{k}$ has to be less than $80^{\circ}$ for the spatially neighboring amino acid $k$ to be considered a functional residue to the asparagine on the $\mathrm{N}$-linked glycosylation [17, 44].

\section{Case study of PTM sites associated with drug binding}

More than 1100 PTM substrate sites related to drug binding were curated and archived in CruxPTM after a large-scale screening for PTM substrate sites and drugbinding sites in the PDB. The number of drug binding associated sites for each PTM type can be found in Table 2. Most of the drug binding sites appeared to be able to undergo phosphorylation, while the second most common PTM among the drug binding sites seemed to be ubiquitylation. According to dbPTM [6], the phosphorylation state of Ser843, situated close to the drug binding site (6.4 $\AA$ ), could influence the affinity of binding for the agonist and inhibitor of the mineralocorticoid receptor (MCR). This is supported by the observation that posphorylated Ser843 reduces the MCR's binding affinity for its agonist and leads to the receptor's own inactivation [45]. Phosphorylation does not always inhibit the protein's activity. In the case of most kinases, while reducing the affinity between a drug and its target, phosphorylation can actually increase the activity of the target protein [11, 46-48]. The insulin-like growth factor 1 receptor (IGF-1R) is an example of the type of kinases (Fig. 3). A case study of IGF-1R shows that an inhibitor of the receptor could maintain the protein in an inactive conformation; however, if the receptor 


\section{a Spatial amino acid composition}

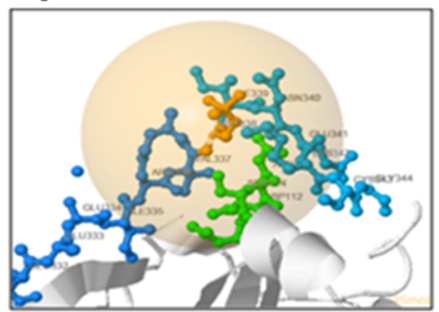

Calculate range $2 \sim 12 \AA$ surround PTM site

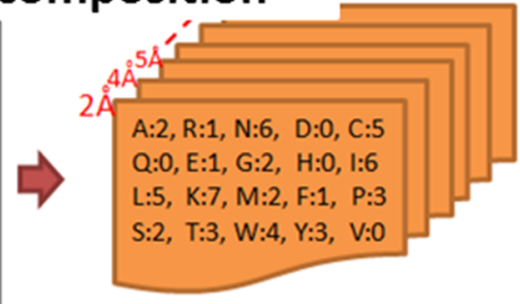

Count 20 Amino Acid in each range

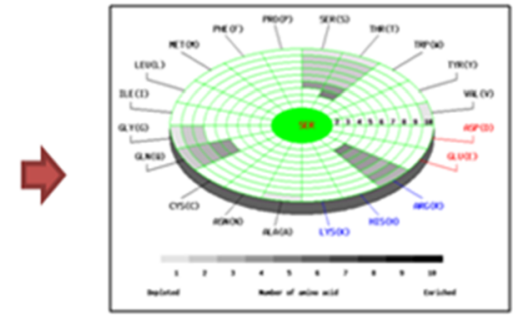

Divide by total residue number of each range and generate composition plot

\section{b Side chain orientation}

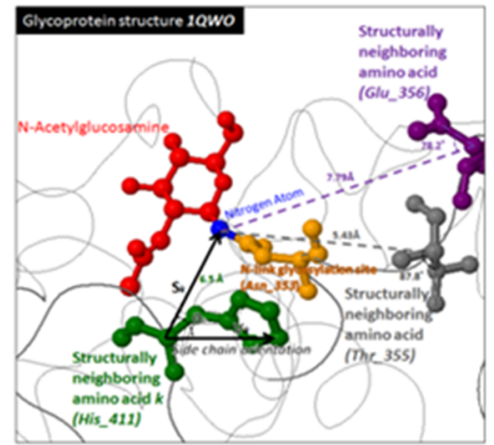

The side chain orientations of $\mathrm{N}$-linked glycosylation substrate site and its spatially neighboring amino acid.

$$
\begin{aligned}
& S_{k}=X_{p}^{S G}-X_{k}^{C \alpha} \\
& V_{k}=X_{k}^{F}-X_{k}^{C \alpha} \\
& \theta_{k}=\arccos \frac{S_{k} \cdot V_{k}}{\left\|S_{k}\right\|\left\|V_{k}\right\|}
\end{aligned}
$$

\section{Secondary structure of flanking sequence}

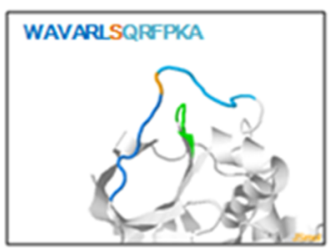

DSSP profile

$G=3-$ turn helix (3, helix). Min length 3 residues.

$H=4$-turn helix $(\underline{\alpha \text { helix }})$. Min length 4 residues.

I= 5 -turn helix ( $\pi$ helix). Min length 5 residues.

$T=$ hydrogen bonded turn $(3,4$ or 5 turn)

$E=$ extended strand in parallel and/or anti-parallel B-sheet conformation.

$B=$ residue in isolated $\beta$-bridge

$S=$ bend (the only non-hydrogen-bond based assignment).

$\mathrm{C}=$ coil (residues which are not in any of the above conformations).

d Division of Acidity and Alkaline residues
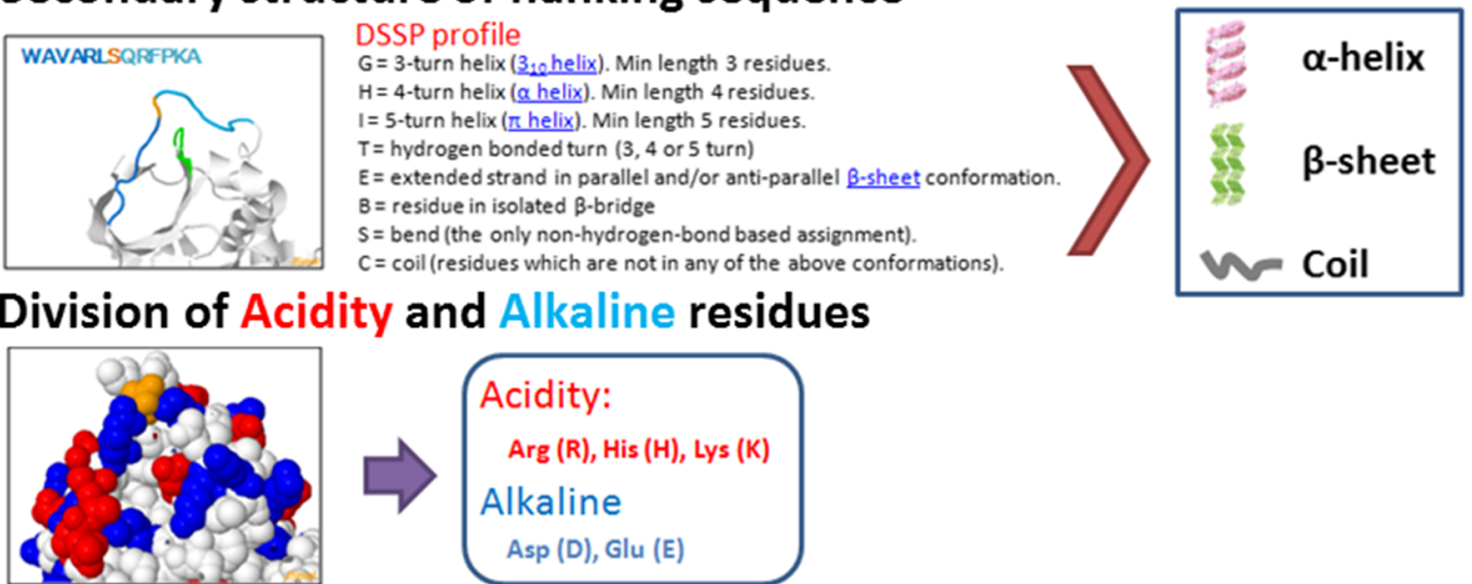

\section{e Solvent-accessible surface area}
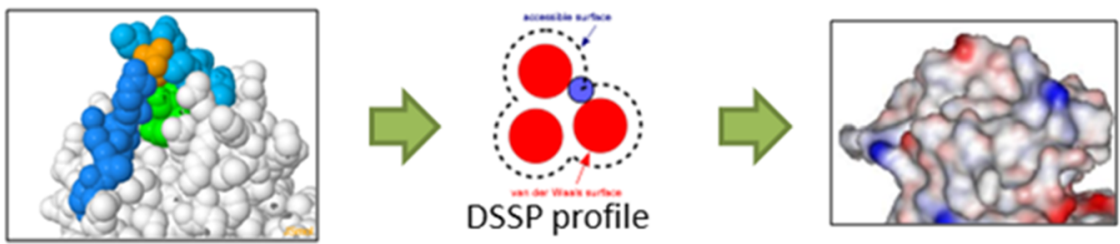

Fig. 2 Investigation of the five structural characteristics for PTM substrate sites. To characterize PTM substrate sites, the structural characteristics such as (a) spatial amino acid composition, (b) the orientation of side chains around PTM substrate sites, (c) secondary structure of flanking sequences, (d) division of acidity and alkaline residues, and (e) solvent-accessible surface area were investigated 
Table 2 Number of PTM sites associated with drug binding sites

\begin{tabular}{|c|c|c|}
\hline PTM Instances & $\begin{array}{l}\text { Number of PTM } \\
\text { sites located on } \\
\text { drug contact sites }\end{array}$ & $\begin{array}{l}\text { Number of PTM sites } \\
\text { within } 12 \AA \text { distance } \\
\text { of drug contact sites }\end{array}$ \\
\hline Phosphorylation & 400 & 1032 \\
\hline Ubiquitylation & 164 & 331 \\
\hline Acetylation & 113 & 296 \\
\hline Pyridoxal phosphate & 84 & 103 \\
\hline N-linked Glycosylation & 82 & 375 \\
\hline O-linked Glycosylation & 51 & 50 \\
\hline S-nitrosylation & 38 & 47 \\
\hline Methylation & 25 & 92 \\
\hline Disulfide bond & 18 & 34 \\
\hline FAD & 17 & 0 \\
\hline Gamma-carboxyglutamic acid & 17 & 32 \\
\hline Proteolytic Cleavage & 17 & 68 \\
\hline C-linked Glycosylation & 15 & 23 \\
\hline N6-carboxylysine & 15 & 12 \\
\hline Carboxylation & 12 & 30 \\
\hline Nucleotide-binding & 8 & 5 \\
\hline Glutathionylation & 6 & 0 \\
\hline Myristoylation & 6 & 10 \\
\hline N6-succinyllysine & 6 & 21 \\
\hline Palmitoylation & 6 & 26 \\
\hline Dephosphorylation & 5 & 5 \\
\hline Oxidation & 5 & 12 \\
\hline FMN & 4 & 0 \\
\hline Prenylation & 4 & 3 \\
\hline Thioether bond & 4 & 19 \\
\hline TPQ & 4 & 0 \\
\hline Deamidation & 3 & 0 \\
\hline Dehydroxylation & 3 & 0 \\
\hline Glycation & 3 & 0 \\
\hline Isopeptide bond & 3 & 0 \\
\hline Neddylation & 3 & 0 \\
\hline Pyruvate & 3 & 0 \\
\hline Sumoylation & 3 & 47 \\
\hline Tryptophylquinone & 3 & 0 \\
\hline ADP-ribosylation & 2 & 3 \\
\hline Biotin & 2 & 0 \\
\hline Deacetylation & 2 & 2 \\
\hline Hydroxylation & 2 & 13 \\
\hline Lipoprotein & 2 & 0 \\
\hline Nitration & 2 & 10 \\
\hline Phosphopantetheine & 2 & 0 \\
\hline Pyrrolidone carboxylic acid & 2 & 21 \\
\hline
\end{tabular}

Table 2 Number of PTM sites associated with drug binding sites (Continued)

\begin{tabular}{lll}
\hline PTM Instances & $\begin{array}{l}\text { Number of PTM } \\
\text { sites located on } \\
\text { drug contact sites }\end{array}$ & $\begin{array}{l}\text { Number of PTM sites } \\
\text { within } 12 \text { A distance } \\
\text { of drug contact sites }\end{array}$ \\
\hline $\begin{array}{l}\text { Tryptophyl-tyrosyl- } \\
\text { methioninium }\end{array}$ & 2 & 0 \\
Allysine & 1 & 0 \\
Amidation & 1 & 36 \\
Carbamidation & 1 & 0 \\
Chromophore & 1 & 0 \\
Formylation & 1 & 1 \\
Lipoyl & 1 & 0 \\
N6-malonyllysine & 1 & 0 \\
S-linked Glycosylation & 1 & 0 \\
Sulfation & 1 & 3 \\
Transglutamination & 1 & 0 \\
TTQ & 1 & 0 \\
Total & 1189 & 2762
\end{tabular}

becomes phosphorylated, the crystal structure of its activation loops would be rearranged in such a way that significantly decreases the inhibitor's affinity for the receptor while enhancing the activity of the receptor. Therefore, phosphorylation may affect the efficacy of a drug by modulating the structure of the target protein and reducing the affinity between the drug and the target.

With reference to a case study discussed on dbPTM, an acetylation site (Lys199) on human serum albumin (HSA) is essential for drug transport and metabolism [49]. Annotations on the OMIM database [50] implicates HSA in hyperthyroxinemia (OMIM ID: 615,999) and analbuminemia (OMIM ID: 616,000). Accordingly, this investigation shows that the Lys199 residue is $4.3 \AA$ from the salicylic acid (DrugBank ID: DB00936) binding site. Aspirin (DrugBank ID: DB00945) has been found to be able to acetylate Lys199, while being hydrolyzed into salicylic acid by HAS [6]. Thus, our investigation confirmed the conformational plasticity of HSA and provided a possible explanation for the regulation of HSAdrug interaction through PTM at the Lys199 residue. Figure 4 shows another example of the modulation of drug-target interaction through PTM. Urease is responsible for hydrolyzing urea into carbon dioxide and ammonia. Carbaoxylation of the Lys 217 residue on the Klebsiella aerogenes urease coordinates the contact between two nickel ions and the drug molecule acetohydroxamic Acid (DrugBank ID: DB00551). The active site of all known ureases is composed of a bis- $\mu$-hydroxo dimeric nickel center, located in the alpha $(\alpha)$-subunit, and has an interatomic distance of $\sim 3.5 \AA$ [51]. Our analysis 


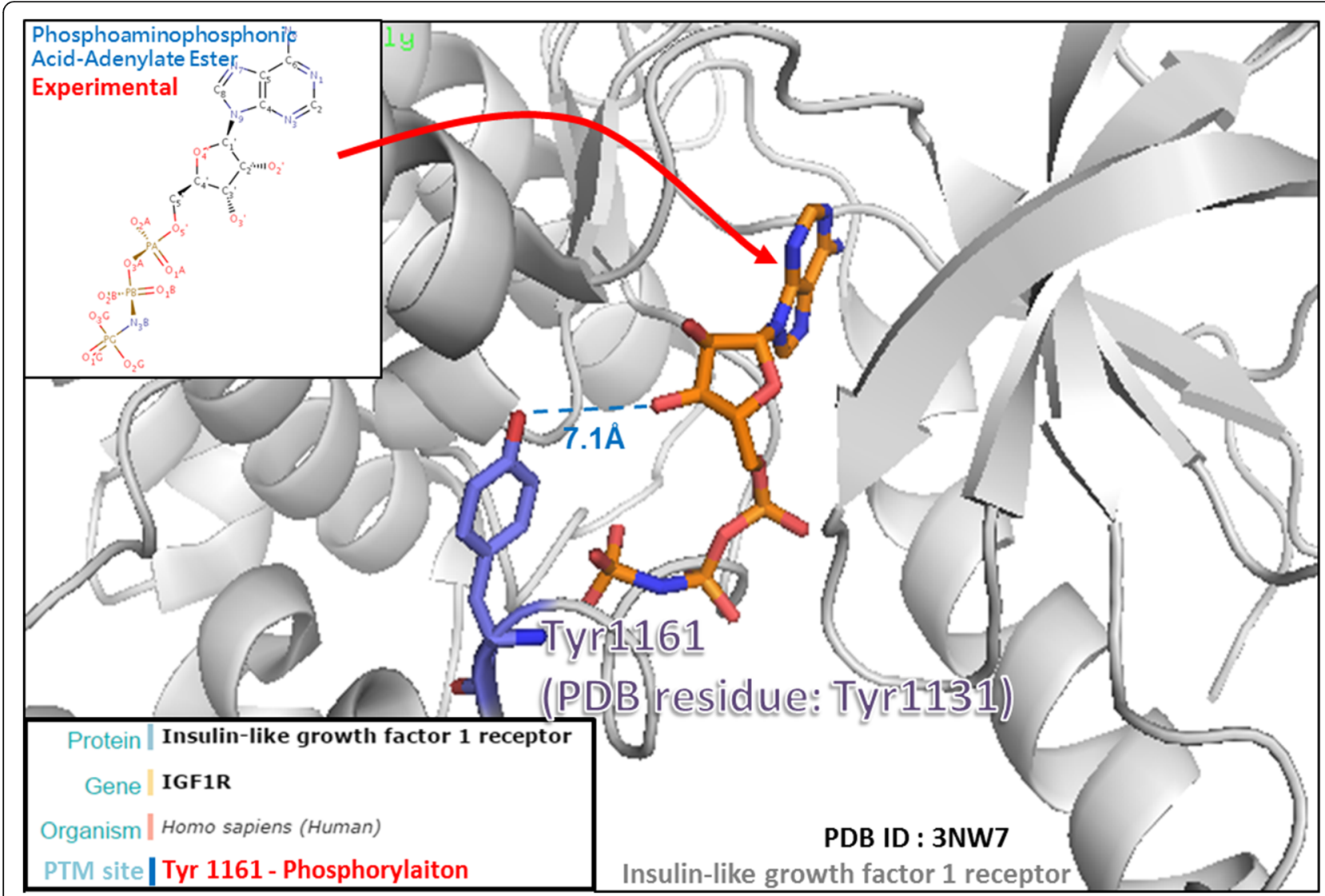

Fig. 3 A case study of the Tyr1131 phosphorylation site associated with drug binding on insulin-like growth factor 1 receptor (IGF1R). The IGF1R is a type of kinases and an inhibitor of the IGF1R could maintain the protein in an inactive conformation. Since the IGF1R was phosphorylated, the crystal structure of its activation loops would be rearranged in such a way that significantly decreases the inhibitor's affinity. Thus, Tyr1131 phosphorylation site may provide a functional role by modulating the structure of the target protein and reducing the affinity between the inhibitor and the target site

shows that acetohydroxamic acid might inhibit urease activity by competing with nickel atoms in the enzyme to form a chelate. This could potentially interrupt the hydrolysis of urea, which reduces the concentration of urinary ammonia and lowers urine $\mathrm{pH}$.

\section{Functions of PTM sites on protein-protein interactions}

As shown in (Additional file 3: Table S3), of all the experimentally verified PTM sites, over $20 \%$ can be found in the functional domains of proteins, implicating the biological significance of PTMs. We studied these sites to infer the roles that these PTMs play in PPI interactions. For instance, approximately $70 \%$ of known $S$-nitrosylation sites, which are responsible for the regulation of NO-related cellular processes, are located in functional domains. Also, among the data that we have collected for the current study, more than 1900 PTM sites are localized to the interface of domain-domain interacting regions. Based on our observations, it appears that structural associations exist between many
PTM sites and binding sites for specific PPI domains and perhaps even regulate the interactions between proteins by modifying the sites of contact.

Cyclin-dependent kinase inhibitor p21, by binding to cyclin-dependent kinases (CDKs), acts as an important checkpoint in cell cycle arrest in response to DNA damage [52]. It can also bind to proliferating cell nuclear antigen (PCNA) to suppress DNA replication [53]. While in solution, the p21 protein does not exhibit a stable structure. However, upon binding to target proteins, the protein assumes an ordered stable conformation. Figure 5 shows that phosphorylation of the Thr145 residue of p21, which corresponds to the PCNA binding region (from residue 144 to 151), may inhibit the interaction between p21 and PCNA, resulting in PCNA binding with other DNA polymerase components [54].

The Rho GDP-dissociation inhibitor 1 (RhoGDI1) is a regulator of the Rho family GTPase [55]. By preventing the release of GDP and the loading of GTP on Rho proteins, RhoGDIs can inhibit the activity of Rho family 


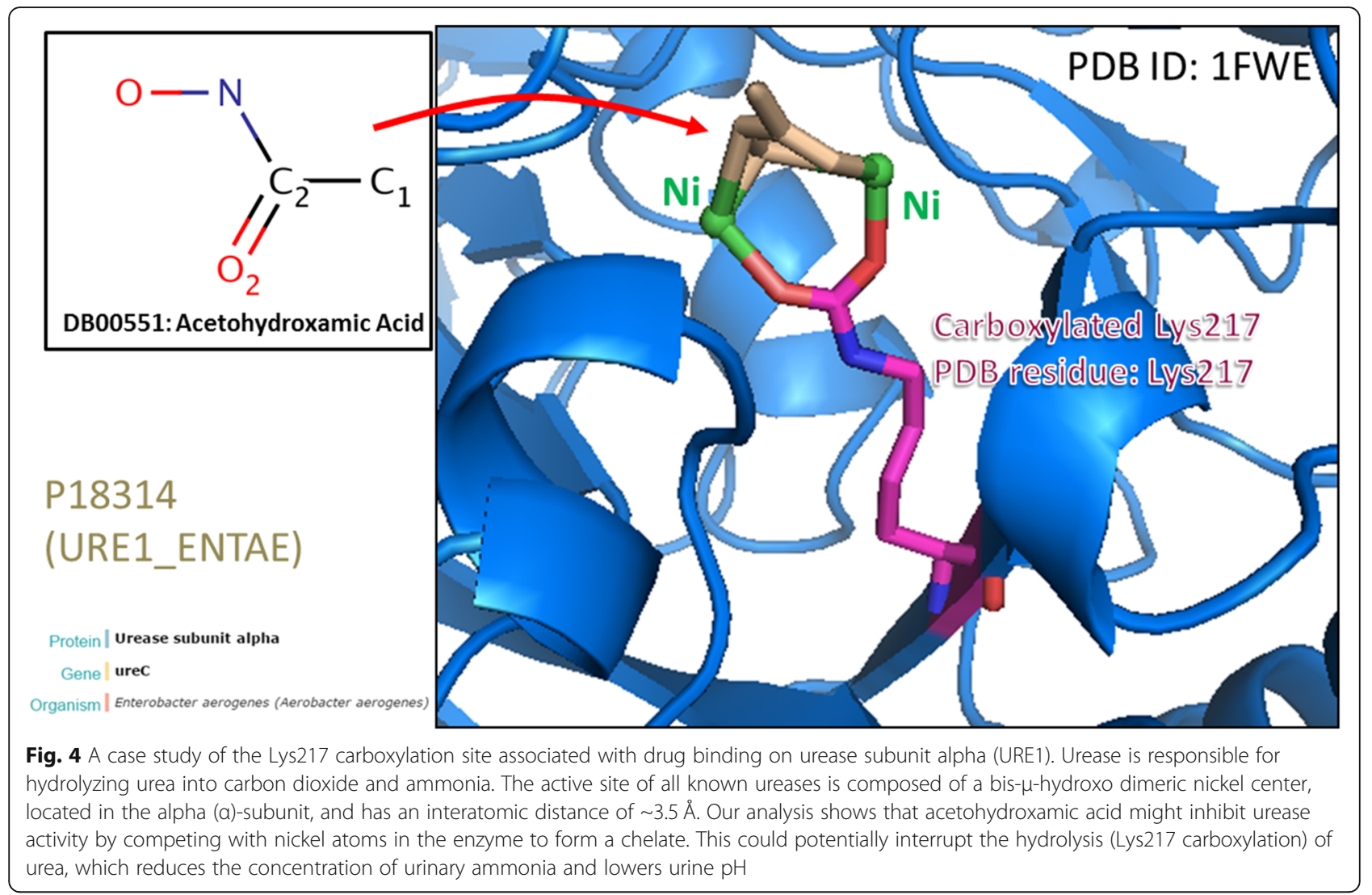

GTPase. RhoGDIs can also block the degradation or improper activation of inactive Rho proteins by transferring them to the cell membrane. The function of RhoGDIs is regulated by phosphorylation at their Ser, Thr and Tyr residues. In fact, phosphorylation at multiple sites in

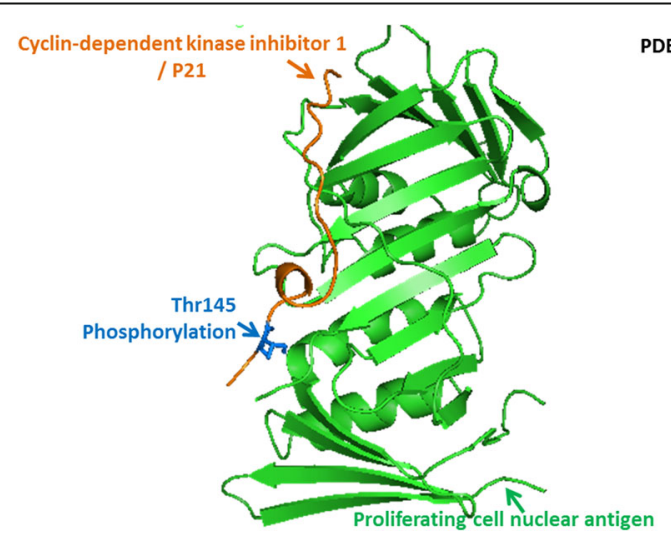

Fig. 5 A case study of the Thr145 phosphorylation site located in the interacting region of p21-PCNA complex (PDBID: 1AXC). The phosphorylation of the Thr145 residue of p21, which corresponds to the PCNA binding region (from residue 144 to 151), may inhibit the interaction between $\mathrm{p} 21$ and PCNA, resulting in PCNA binding with other DNA polymerase components [54]
RhoGDIs can stimulate the simultaneous release of multiple Rho proteins [55]. The key functional region of RhoGDIs lies in its $\mathrm{N}$-terminal domain. This region, though generally disordered, can form two helices and bind to the switch I and switch II regions of GTPase to prohibit the latter from making conformational changes required for the exchange of GDP and GTP [56]. As presented in Fig. 6, the disordered N-terminal domain of RhoGDI1 contains a Tyr residue (Tyr27), which is localized to the binding interface and can be phosphorylated to facilitate the dissociation of RhoA, Rac1, and cdc from RhoGDI1, making GTPases available for activation [57].

The crystal structure of the ternary complex of the eIF4E-m7GpppA-4EBP1 peptide is shown in Fig. 7. This structure, formed from the interaction among the 7 methylguanosine at the $5^{\prime}$-cap of mature transcripts, eukaryotic initiation factor 4E (eIF4E), and endogenous $4 \mathrm{E}$-binding protein 1 (4EBP1), is required for translation initiation [58]. Figure 7 indicates that three substrate sites (Thr50, Tyr54 and Ser65) of phosphorylation are located within the binding region of the 4EBP1, which can regulate its interaction with eIF4E. The three sites are reported to modulate the reversible binding of 4EBP1 with eIF4E, and hyper-phosphorylation at these sites can decrease the strength of interaction between the two proteins [59]. 


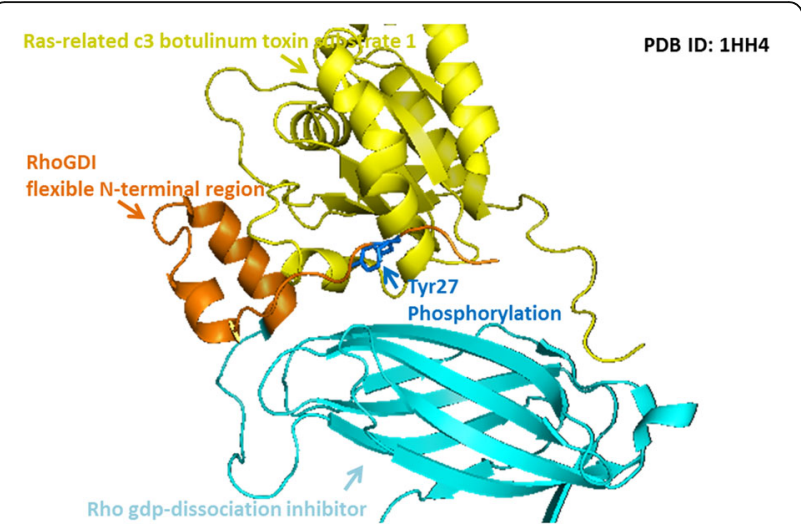

Fig. 6 A case study of the Tyr127 phosphorylation site located in the interacting region of RhoGDI-Rac1 complex (PDBID: 1HH4). A disordered $\mathrm{N}$-terminal domain of RhoGDI1 contains a tyrosine residue (Tyr27), which is localized to the docking interface. The phosphorylated Tyr127 has been reported to facilitate the dissociation of RhoA, Rac1, and cdc from RhoGDI1, making GTPases available for activation [57]

\section{Conclusion}

In this study, we first mapped PTM sites to the 3D structures of proteins, and adopted multiple methods to describe the structural characteristics of PTM sites in tertiary structures. Already, studies are emerging that use similar methods to investigate PTM; for example, Karabulut and Frishman's study [60] that utilizes spatial amino acid composition to identify acetylation sites. However, by employing several different approaches and considering several structural characteristics of a variety of PTM sites associated with drug-target binding and PPI, this work can effectively facilitate the functional

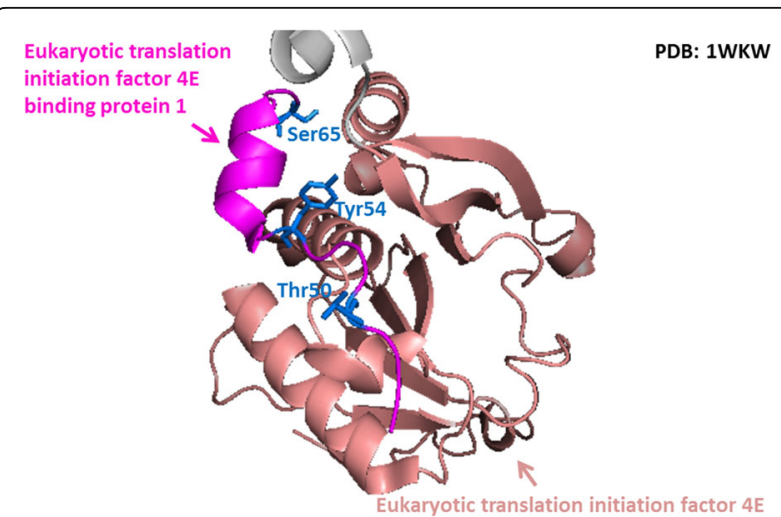

Fig. 7 A case study of the phosphorylation sites located in the interacting region of the ternary complex of elF4E-m7GpppA-4EBP1 peptide (PDBID: 1WKW). There are three substrate sites (Thr50, Tyr54 and Ser65) of phosphorylation within the binding region of the $4 \mathrm{EBP} 1$, which can regulate its interaction with elF4E. These sites are reported to modulate the reversible binding of 4EBP1 with elF4E, and hyper-phosphorylation at these sites decreases the strength of interaction between the two proteins [59] study of various types of PTM. Indeed, the reliability of our analysis can be supported by the fact that other studies also identified some of the drug-binding and PPI associated PTM sites uncovered in our investigation.

Our approach has the potential to be applied on drug design, which often centers around the influence of amino acid mutation on the effect of a drug. However, PTMs are also affected by changes in the amino acid sequence. Our study indicates that PTMs can be crucial to a drug's effect on a structural level, and knowing PTM sites associated with protein-protein interaction is helpful for understanding the biological mechanisms involving these PTM sites.

For situations where information regarding the protein's structure is lacking, we attempted to overcome this limitation with molecular docking. According to the latest statistics from the PDB in 2016, there are over 122,000 records for protein structures. Although the number of annotated PDB structures is increasing rapidly, information of structural proteins is still limited. When cross-referenced with annotations on UniProtKB, it was found that only 23,605 out of 551,705 reviewed proteins and 12,165 out of 114,895 PTM proteins have crystal structure information, respectively. Some proteins only have partially annotated crystal structure related to specific fragments in their sequences such that it was impossible to map the PTM sites to these proteins' 3D structures. For example, the ankyrin-3 protein have 16 experimental and 17 putative PTM sites within its sequence of 4377 amino acids, but only the region between amino acid 4088 and 4199 has annotated crystal structure. As a result, only one PTM site could be mapped to this structure. This limitation may affect the reliability of comparison among PTM sites.

CruxTPM is a novel, integrative web platform for the analysis of PTMs and their biological roles in a 3D structural context. It enables the structural characterization and 3D visualization of PTM sites, as well as the investigation of their relationship with drug-target binding and PPI. The tool also provides interactive function like drug structure search, PTM modified structure visualization, online small molecule docking, etc. We hope this study and analytical platform can help enhance the understanding of the biological mechanisms associated with PTMs and improve the efficiency of drug design.

\section{Additional files}

Additional file 1: Table S1. Classification and definition of drugs. (PDF $70 \mathrm{~kb}$ )

Additional file 2: Table S2. Number of PTM sites that can be mapped to the PDB 3D structures. (PDF $41 \mathrm{~kb}$ )

Additional file 3: Table S3. Number of PTM sites located in proteinprotein interaction regions. (PDF $155 \mathrm{~kb}$ ) 


\section{Acknowledgements}

Not applicable.

\section{Funding}

Publication charge for this work was funded by the Ministry of Science and Technology (MOST) of Taiwan under contract numbers of MOST 104-2221-E155-036-MY2 and MOST 106-2221-E-155-063 to TYL.

\section{Availability of data and materials}

The datasets used and analysed during the current study available from the corresponding author on reasonable request.

\section{About this supplement}

This article has been published as part of BMC Systems Biology Volume 11 Supplement 7, 2017: 16th International Conference on Bioinformatics (InCoB 2017): Systems Biology. The full contents of the supplement are available online at https://bmcsystbiol.biomedcentral.com/articles/supplements/ volume-11-supplement-6.

\section{Authors' contributions}

TYL and JTYW conceived and designed the experiments. MGS, JBKH, and YHC performed the experiments. MGS and KYH analyzed the data. MGS and JBKH wrote the manuscript with revision by TYL and JTYW. All authors read and approved the final manuscript.

\section{Ethics approval and consent to participate}

Not applicable.

\section{Consent for publication}

Not applicable.

\section{Competing interests}

The authors declare that they have no competing interests.

\section{Publisher's Note}

Springer Nature remains neutral with regard to jurisdictional claims in published maps and institutional affiliations.

\section{Author details}

'Department of Computer Science and Engineering, Yuan Ze University, Taoyuan 320, Taiwan. ${ }^{2}$ Department of Medical Research, Taipei Medical University Hospital, Taipei 110, Taiwan. ${ }^{3}$ Department of Medical Research, Hsinchu Mackay Memorial Hospital, Hsinchu City 300, Taiwan. ${ }^{4}$ Innovation Center for Big Data and Digital Convergence, Yuan Ze University, Taoyuan 320, Taiwan

\section{Published: 21 December 2017}

\section{References}

1. Lander ES, Linton LM, Birren B, Nusbaum C, Zody MC, Baldwin J, Devon K, Dewar K, Doyle M, FitzHugh W, et al. Initial sequencing and analysis of the human genome. Nature. 2001;409(6822):860-921.

2. van Kasteren SI, Kramer HB, Jensen HH, Campbell SJ, Kirkpatrick J, Oldham NJ, Anthony DC, Davis BG. Expanding the diversity of chemical protein modification allows post-translational mimicry. Nature. 2007;446(7139):1105-9.

3. Lee TY, Huang HD, Hung JH, Huang HY, Yang YS, Wang TH. dbPTM: an information repository of protein post-translational modification. Nucleic Acids Res. 2006:34(Database issue):D622-7.

4. Lee TY, Bo-Kai Hsu J, Chang WC, Huang HD. RegPhos: a system to explore the protein kinase-substrate phosphorylation network in humans. Nucleic Acids Res. 2011;39(Database issue):D777-87.

5. Seet BT, Dikic I, Zhou MM, Pawson T. Reading protein modifications with interaction domains. Nat Rev Mol Cell Biol. 2006;7(7):473-83.

6. Huang KY, Su MG, Kao HJ, Hsieh YC, Jhong JH, Cheng KH, Huang HD, Lee TY. dbPTM 2016: 10-year anniversary of a resource for post-translational modification of proteins. Nucleic Acids Res. 2016;44(D1):D435-46.

7. Lu CT, Huang KY, Su MG, Lee TY, Bretana NA, Chang WC, Chen YJ, Huang HD. DbPTM 3.0: an informative resource for investigating substrate site specificity and functional association of protein post-translational modifications. Nucleic Acids Res. 2013;41(Database issue):D295-305.
8. Nguyen VN, Huang KY, Weng JT, Lai KR, Lee TY. UbiNet: an online resource for exploring the functional associations and regulatory networks of protein ubiquitylation. Database (Oxford). 2016;2016

9. Nguyen TD, Vidal-Cortes O, Gallardo O, Abian J, Carrascal M. LymPHOS 2.0 an update of a phosphosite database of primary human T cells. Database (Oxford). 2015:2015.

10. Chen YJ, Lu CT, Su MG, Huang KY, Ching WC, Yang HH, Liao YC, Chen YJ, Lee TY. dbSNO 2.0: a resource for exploring structural environment, functional and disease association and regulatory network of protein Snitrosylation. Nucleic Acids Res. 2015;43(Database issue):D503-11.

11. Huang KY, Wu HY, Chen YJ, Lu CT, Su MG, Hsieh YC, Tsai CM, Lin Kl, Huang HD, Lee TY, et al. RegPhos 2.0: an updated resource to explore protein kinase-substrate phosphorylation networks in mammals. Database (Oxford). 2014;2014(0):bau034

12. Lee TY, Chen YJ, CT L, Ching WC, Teng YC, Huang HD, Chen YJ. dbSNO: a database of cysteine S-nitrosylation. Bioinformatics. 2012;28(17):2293-5.

13. Chernorudskiy AL, Garcia A, Eremin EV, Shorina AS, Kondratieva EV, Gainullin MR. UbiProt: a database of ubiquitylated proteins. BMC Bioinformatics. 2007; 8:126.

14. Hornbeck PV, Chabra I, Kornhauser JM, Skrzypek E, Zhang B. PhosphoSite: a bioinformatics resource dedicated to physiological protein phosphorylation. Proteomics. 2004;4(6):1551-61.

15. Zanzoni A, Ausiello G, Via A, Gherardini PF, Helmer-Citterich M. Phospho3D: a database of three-dimensional structures of protein phosphorylation sites. Nucleic Acids Res. 2007;35(Database issue):D229-31.

16. Zanzoni A, Carbajo D, Diella F, Gherardini PF, Tramontano A, HelmerCitterich M, Via A. Phospho3D 2.0: an enhanced database of threedimensional structures of phosphorylation sites. Nucleic Acids Res. 2011; 39(Database issue):D268-71.

17. Su MG, Lee TY. Incorporating substrate sequence motifs and spatial amino acid composition to identify kinase-specific phosphorylation sites on protein three-dimensional structures. BMC Bioinformatics. 2013;14(Suppl 16):S2.

18. Su MG, Huang KY, Lu CT, Kao HJ, Chang YH, Lee TY. topPTM: a new module of dbPTM for identifying functional post-translational modifications in transmembrane proteins. Nucleic Acids Res. 2014;42(Database issue):D537-45.

19. Craveur P, Rebehmed J, de Brevern AG. PTM-SD: a database of structurally resolved and annotated posttranslational modifications in proteins. Database (Oxford). 2014:2014

20. The UniProt C. UniProt: the universal protein knowledgebase. Nucleic Acids Res. 2017:45(D1):D158-69.

21. Woodford MR, Dunn D, Miller JB, Jamal S, Neckers L, Mollapour M. Impact of posttranslational modifications on the anticancer activity of Hsp90 inhibitors. Adv Cancer Res. 2016;129:31-50.

22. Mahalingam D, Swords R, Carew JS, Nawrocki ST, Bhalla K, Giles FJ. Targeting HSP90 for cancer therapy. Br J Cancer. 2009:100(10):1523-9.

23. Leonard PG, Golemi-Kotra D, Stock AM. Phosphorylation-dependent conformational changes and domain rearrangements in Staphylococcus Aureus VraR activation. Proc Natl Acad Sci U S A. 2013;110(21):8525-30.

24. Smith KP, Gifford KM, Waitzman JS, Rice SE. Survey of phosphorylation near drug binding sites in the protein data Bank (PDB) and their effects. Proteins. 2015:83(1):25-36.

25. McCubrey JA, Steelman LS, Chappell WH, Abrams SL, Wong EW, Chang F, Lehmann B, Terrian DM, Milella M, Tafuri A, et al. Roles of the Raf/MEK/ERK pathway in cell growth, malignant transformation and drug resistance. Biochim Biophys Acta. 2007:1773(8):1263-84.

26. Yazdanian M, Briggs K, Jankovsky C, Hawi A. The "high solubility" definition of the current FDA guidance on biopharmaceutical classification system may be too strict for acidic drugs. Pharm Res. 2004;21(2):293-9.

27. Gavin AC, Bosche M, Krause R, Grandi P, Marzioch M, Bauer A, Schultz J, Rick JM Michon AM, Cruciat CM, et al. Functional organization of the yeast proteome by systematic analysis of protein complexes. Nature. 2002;415(6868):141-7.

28. Evidence for network evolution in an Arabidopsis interactome map. Science. 2011;333(6042):601-7.

29. De Las Rivas J, Fontanillo C. Protein-protein interaction networks: unraveling the wiring of molecular machines within the cell. Brief Funct Genomics. 2012:11(6):489-96.

30. Nishi H, Hashimoto K, Panchenko AR. Phosphorylation in protein-protein binding: effect on stability and function. Structure. 2011:19(12):1807-15.

31. Vinayagam A, Stelzl U, Foulle R, Plassmann S, Zenkner M, Timm J, Assmus HE, Andrade-Navarro MA, Wanker EE. A directed protein interaction network for investigating intracellular signal transduction. Sci Signal. 2011;4(189):rs8. 
32. De Las Rivas J, Fontanillo C. Protein-protein interactions essentials: key concepts to building and analyzing interactome networks. PLoS Comput Biol. 2010;6(6):e1000807.

33. Mosca R, Ceol A, Stein A, Olivella R, Aloy P. 3did: a catalog of domain-based interactions of known three-dimensional structure. Nucleic Acids Res. 2014; 42(Database issue):D374-9.

34. Rose PW, Prlic A, Altunkaya A, Bi C, Bradley AR, Christie CH, Costanzo LD, Duarte JM, Dutta S, Feng Z, et al. The RCSB protein data bank: integrative view of protein, gene and 3D structural information. Nucleic Acids Res. 2017:45(D1):D271-81.

35. Law V, Knox C, Djoumbou Y, Jewison T, Guo AC, Liu Y, Maciejewski A, Arndt D, Wilson M, Neveu V, et al. DrugBank 4.0: shedding new light on drug metabolism. Nucleic Acids Res. 2014:42(Database issue):D1091-7.

36. KSaM R. PoseView - molecular interaction patterns at a glance. Journal of Cheminformatics. 2010;2(Suppl 1)

37. Hsu KC, Chen YF, Lin SR, Yang JM. iGEMDOCK: a graphical environment of enhancing GEMDOCK using pharmacological interactions and postscreening analysis. BMC Bioinformatics. 2011;12(Suppl 1):S33.

38. Aloy P, Russell RB. Interrogating protein interaction networks through structural biology. Proc Natl Acad Sci U S A. 2002;99(9):5896-901.

39. Shien DM, Lee TY, Chang WC, Hsu JB, Horng JT, Hsu PC, Wang TY, Huang HD. Incorporating structural characteristics for identification of protein methylation sites. J Comput Chem. 2009;30(9):1532-43.

40. Kabsch W, Sander C. Dictionary of protein secondary structure: pattern recognition of hydrogen-bonded and geometrical features. Biopolymers. 1983;22(12):2577-637.

41. Lee TY, Chen YJ, TC L, Huang HD, Chen YJ. SNOSite: exploiting maximal dependence decomposition to identify cysteine S-nitrosylation with substrate site specificity. PLoS One. 2011;6(7):e21849.

42. Durek P, Schudoma C, Weckwerth W, Selbig J, Walther D. Detection and characterization of 3D-signature phosphorylation site motifs and their contribution towards improved phosphorylation site prediction in proteins. BMC Bioinformatics. 2009;10

43. Herraez A. Biomolecules in the computer - Jmol to the rescue. Biochem Mol Biol Edu. 2006;34(4):255-61.

44. Chien YT, Huang SW. Accurate prediction of protein catalytic residues by side chain orientation and residue contact density. PLoS One. 2012;7(10): e47951.

45. Salwinski L, Miller CS, Smith AJ, Pettit FK, Bowie JU, Eisenberg D. The database of interacting proteins: 2004 update. Nucleic Acids Res. 2004; 32(Database issue):D449-51.

46. Lee TY, Bretana NA, CT L. PlantPhos: using maximal dependence decomposition to identify plant phosphorylation sites with substrate site specificity. BMC Bioinformatics. 2011;12:261.

47. Wong YH, Lee TY, Liang HK, Huang CM, Wang TY, Yang YH, Chu CH, Huang HD, Ko MT, Hwang JK. KinasePhos 2.0: a web server for identifying protein kinase-specific phosphorylation sites based on sequences and coupling patterns. Nucleic Acids Res. 2007;35(Web Server issue):W588-94.

48. Huang HD, Lee TY, Tzeng SW, Horng JT. KinasePhos: a web tool for identifying protein kinase-specific phosphorylation sites. Nucleic Acids Res. 2005;33(Web Server issue):W226-9.

49. Licata L, Briganti L, Peluso D, Perfetto L, lannuccelli M, Galeota E, Sacco F, Palma A, Nardozza AP, Santonico E, et al. MINT, the molecular interaction database: 2012 update. Nucleic Acids Res. 2012;40(Database issue):D857-61.

50. Amberger JS, Bocchini CA, Schiettecatte F, Scott AF, Hamosh A. OMIM.org: online Mendelian inheritance in man $(\mathrm{OMIM}(\mathrm{R}))$, an online catalog of human genes and genetic disorders. Nucleic Acids Res. 2015;43(Database issue):D789-98.

51. Moncrief MB, Hom LG, Jabri E, Karplus PA, Hausinger RP. Urease activity in the crystalline state. Protein Sci. 1995;4(10):2234-6.

52. Neganova I, Vilella F, Atkinson SP, Lloret M, Passos JF, von Zglinicki T, O'Connor JE, Burks D, Jones R, Armstrong $L$, et al. An important role for CDK2 in $\mathrm{G} 1$ to $S$ checkpoint activation and DNA damage response in human embryonic stem cells. Stem Cells. 2011;29(4):651-9.

53. Gartel AL, Tyner AL. The role of the cyclin-dependent kinase inhibitor p21 in apoptosis. Mol Cancer Ther. 2002;1(8):639-49.

54. Rossig L, Jadidi AS, Urbich C, Badorff C, Zeiher AM, Dimmeler S. Aktdependent phosphorylation of p21(Cip1) regulates PCNA binding and proliferation of endothelial cells. Mol Cell Biol. 2001;21(16):5644-57.

55. Garcia-Mata R, Boulter E, Burridge K. The 'invisible hand': regulation of RHO GTPases by RHOGDIs. Nat Rev Mol Cell Biol. 2011;12(8):493-504.
56. Grizot S, Faure J, Fieschi F, Vignais PV, Dagher MC, Pebay-Peyroula E. Crystal structure of the Rac1-RhoGDI complex involved in nadph oxidase activation Biochemistry. 2001;40(34):10007-13.

57. DerMardirossian C, Rocklin G, Seo JY, Bokoch GM. Phosphorylation of RhoGDI by Src regulates rho GTPase binding and cytosol-membrane cycling. Mol Biol Cell. 2006;17(11):4760-8.

58. Svitkin $\mathrm{Y}$, Herdy B, Costa-Mattioli M, Gingras AC, Raught B, Sonenberg N. Eukaryotic translation initiation factor $4 \mathrm{E}$ availability controls the switch between cap-dependent and internal ribosomal entry site-mediated translation. Mol Cell Biol. 2005;25(23):10556-65.

59. Gingras AC, Gygi SP, Raught B, Polakiewicz RD, Abraham RT, Hoekstra MF, Aebersold R, Sonenberg N. Regulation of 4E-BP1 phosphorylation: a novel two-step mechanism. Genes Dev. 1999;13(11):1422-37.

60. Karabulut NP, Frishman D. Tissue-specific sequence and structural environments of lysine acetylation sites. J Struct Biol. 2015;191(1):39-48.

\section{Submit your next manuscript to BioMed Central and we will help you at every step:}

- We accept pre-submission inquiries

- Our selector tool helps you to find the most relevant journal

- We provide round the clock customer support

- Convenient online submission

- Thorough peer review

- Inclusion in PubMed and all major indexing services

- Maximum visibility for your research

Submit your manuscript at www.biomedcentral.com/submit

) Biomed Central 